\title{
Histopatologia de lesões tumoriformes presentes na cavidade nasal de eqüídeos do Brasil
}

\author{
Histopathology of tumor-like masses present in the nasal cavity of equids from Brazil
}

\author{
Marcele Nogueira de Sousa Trotte ${ }^{\mathrm{I}}$ Isabele Barbieri dos Santos ${ }^{\mathrm{II}}$ \\ Luisa Helena Monteiro de Miranda ${ }^{\text {II }}$ Ariane Ribeiro Amorim ${ }^{\text {II }}$ \\ José Renato Junqueira Borges ${ }^{\mathrm{IV}}$ \\ Rodrigo Caldas Menezes ${ }^{\text {II }}$
}

\section{RESUMO}

Este estudo foi realizado com o objetivo de analisar os aspectos microscópicos e enfatizar a importância do exame histopatológico na determinação do diagnóstico de lesões tumoriformes na cavidade nasal de eqüídeos. Para tanto, foram estudados microscopicamente cortes de tecido de 11 eqüídeos com lesões tumoriformes na cavidade nasal. Essas amostras foram enviadas ao Serviço de Anatomia Patológica da Faculdade de Medicina Veterinária da Universidade Federal Fluminense, Niterói, Rio de Janeiro, no período de 2000 a 2004. Foram diagnosticados três casos de rinosporidiose, um de amiloidose, seis de pólipos nasais e um de hematoma etmoidal progressivo.

Palavras-chave: amiloidose, eqüídeos, hematoma etmoidal, pólipo nasal, rinosporidiose.

\section{ABSTRACT}

The purpose of the present study was to analyze the microscopic aspects of tumor-like masses present in the nasal cavity of equids and also to emphasize the importance of histopathological examination for determining the diagnosis. Tissue sections of tumor-like masses from the nasal cavity of 11 equids were microscopically examined. These samples were sent to the Anatomic Pathology Service of the Veterinary Medicine College from the Fluminense Federal University, Niterói, Rio de Janeiro, from 2000 to 2004. Three cases of rhinosporidiosis, one of amyloidosis, six of nasal polyps and one of progressive ethmoid hematoma were diagnosed.

Key words: amyloidosis, equids, ethmoid hematoma, nasal polyp, rhinosporidiosis.

\section{INTRODUÇÃO}

A obstrução da cavidade nasal por lesões tumoriformes é por vezes encontrada em eqüídeos. O termo pólipo nasal tem sido usado erroneamente para descrever lesões tumoriformes com aspecto pedunculado e polipóide (NICKELS, 1993). Além dos verdadeiros pólipos nasais, que são crescimentos pedunculados de origem inflamatória surgindo da mucosa nasal, cuja etiologia é desconhecida, existem outras causas dessas lesões tumoriformes, como as infecções fúngicas, a rinosporidiose, o hematoma etmoidal, as neoplasias e a amiloidose (STICKLE \& JONES, 1976; NICKELS, 1993; KNOTTENBELT \& PASCOE, 1994; JONES et al., 2000).

A rinosporidiose caracteriza-se por uma lesão granulomatosa tumoriforme causada pelo Rhinosporidium seeberi, particularmente na mucosa nasal (JONES et al., 2000). Já foram relatados casos em cavalos, bovinos, cães, patos, gansos, seres humanos (JONES et al., 2000), gatos (MOISAN \& BAKER, 2001), cisnes (KENNEDY et al., 1995) e mulas (LONDERO et al., 1977; BERROCAL \& LÓPEZ, 2007).

O cavalo é sujeito ao desenvolvimento de amiloidose, que é o depósito de glicoproteínas com estrutura ß-pregueada em vários órgãos (KIM et al., 2005). No trato respiratório, os locais mais usuais para

\footnotetext{
'Departamento de Patologia e Clínica Veterinária, Faculdade de Veterinária, Universidade Federal Fluminense (UFF). Rua vital Brazil Filho, 64, 24230-340, Santa Rosa, Niterói, RJ, Brasil. E-mail: marceletrotte@vm.uff.br. Autor para correspondência. "Serviço de Zoonoses, Instituto de Pesquisa Clínica Evandro Chagas, Fundação Oswaldo Cruz (Fiocruz), Rio de Janeiro, RJ, Brasil. IIICurso de Medicina Veterinária, UFF, Niterói, RJ, Brasil.

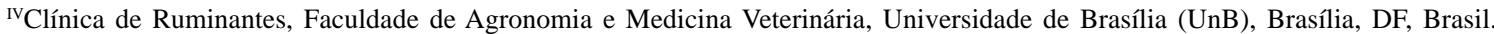


esta deposição são as narinas e a cavidade nasal, principalmente o septo nasal e as conchas nasais. $\mathrm{Na}$ cavidade nasal, a lesão macroscópica da amiloidose é caracterizada pela formação de placas firmes e pálidas (KNOTTENBELT \& PASCOE, 1994).

O hematoma etmoidal progressivo consiste de massas angiomatosas de expansão lenta (PASCOE, 1993). Ele origina-se no revestimento mucoso do osso etmoturbinado ou da parede dos seios maxilares, esfenopalatinos e frontais (TATE, 2002) e ocorre freqüentemente em cavalos entre oito e 10 anos de idade (KNOTTENBELT \& PASCOE, 1994).

Os animais acometidos por patologias na cavidade nasal apresentam sinais clínicos semelhantes, sendo mais comuns a secreção nasal, epistaxe e dispnéia (NICKELS, 1993). Portanto, para o diagnóstico definitivo e diferencial dessas afecções, é necessária a realização de exame histológico (STICKLE \& JONES, 1976; SPECHT et al., 1990; NICKELS, 1993; WATT \& BECK, 1997; BERROCAL \& LÓPEZ, 2007). No caso das micoses, é fundamental também a realização de cultura micológica (NICKELS, 1993).

No Brasil não há relatos de levantamentos da ocorrência e do diagnóstico diferencial das lesões tumoriformes na cavidade nasal de eqüídeos. Portanto, o presente estudo teve por objetivo analisar os aspectos histopatológicos de afecções que causam lesões tumoriformes na cavidade nasal de eqüídeos e enfatizar a importância do exame histopatológico na determinação do diagnóstico definitivo.

\section{MATERIAL E MÉTODOS}

Foram analisados ao microscópio cortes de tecido de 11 eqüídeos (dez cavalos e uma mula) apresentando lesões tumoriformes na cavidade nasal, obtidos nos arquivos do Serviço de Anatomia Patológica da Faculdade de Medicina Veterinária da Universidade Federal Fluminense, Niterói, Rio de Janeiro. A origem dos animais é desconhecida.

As amostras estudadas foram enviadas durante o período de 2000 a 2004 com a finalidade de diagnóstico. Elas foram processadas pelas técnicas histológicas usuais com inclusão em parafina e coradas pela hematoxilina-eosina e pelo vermelho-congo em caso de suspeita de amiloidose. As lâminas foram analisadas em microscópico óptico (Olympus Bx 41) e as fotografias realizadas com câmera digital.

\section{RESULTADOSE DISCUSSÃO}

Dos 11 animais com lesões tumoriformes na cavidade nasal, foram diagnosticados três casos de rinosporidiose, um caso de amiloidose, seis casos de pólipos nasais e um caso de hematoma etmoidal progressivo. Com exceção de um pólipo em uma mula, todas as afecções ocorreram em cavalos. No exame clínico desses animais, as lesões foram relatadas como tumoriformes.

O exame histopatológico dos casos de rinosporidiose revelou hiperplasia do epitélio escamoso estratificado do vestíbulo (Figura 1A). A presença de microrganismos esféricos com parede dupla e espessa (esporângios) contendo endosporos foi observada no epitélio (Figura 1A) e no estroma (Figura 1B). Os endosporos eram maiores e mais uniformemente redondos no centro dos esporângios e apresentavam vários corpos eosinofílicos no seu interior. Os esporângios estavam circundados por inflamação granulomatosa composta de células epitelióides, gigantes multinucleadas tipo Langhans, linfócitos,
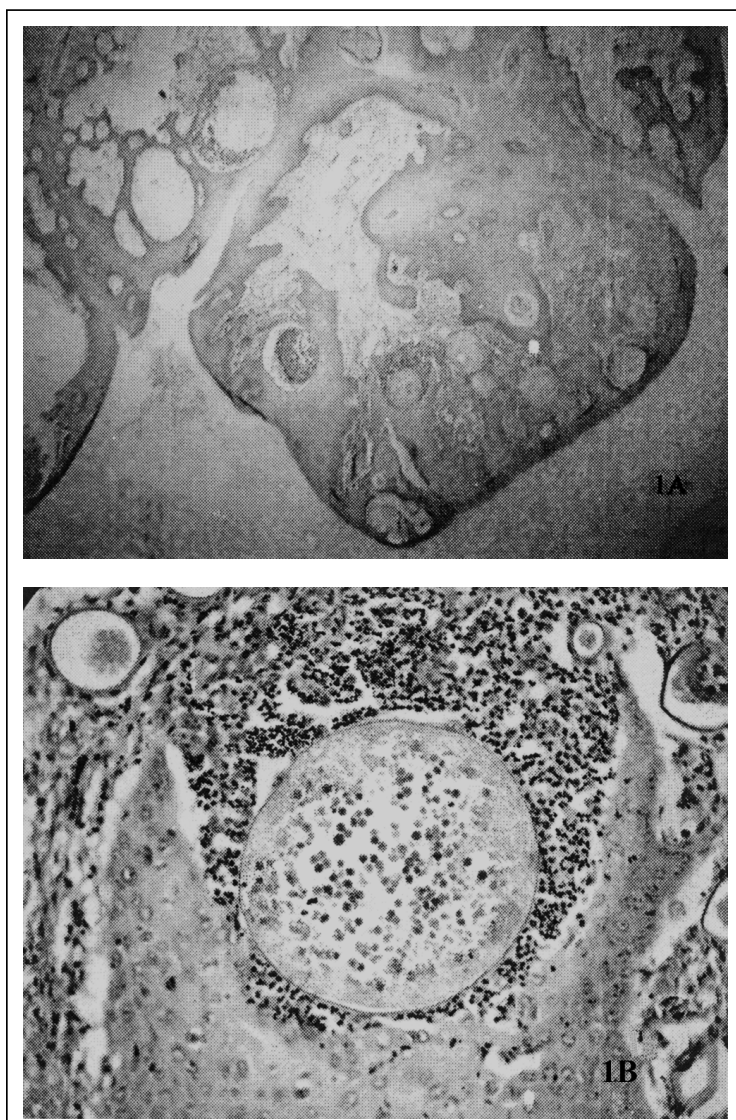

Figura 1 - (A) Cavidade nasal. Rinosporidiose. Égua Puro Sangue Inglês de três anos de idade. Epitélio escamoso estratificado hiperplásico (do vestíbulo) com presença de esporângios em diferentes estágios de maturação. HE, obj. 4X. 1(B)-Detalhe da figura 1 (A), mostrando o esporângio com endosporos no interior e a reação granulomatosa ao redor do esporângio. HE, obj. 40X. 
neutrófilos e tecido conjuntivo fibrovascular. Estes achados estavam de acordo com os descritos por outros autores (LONDERO et al., 1977; JONES et al., 2000; BERROCAL \& LÓPEZ, 2007). O diagnóstico diferencial de coccidioidomicose baseou-se na presença de esporângios e endosporos maiores possuindo corpos eosinofílicos (JONES et al., 2000). A rinosporidiose é uma doença associada a águas estagnadas e é comum na Índia, no Sri Lanka e no sudeste da Ásia (JONES et al.,2000), ao contrário da coccidioidomicose, que ocorre em regiões áridas e semi-áridas do continente americano. No Brasil, as áreas endêmicas da coccidioidomicose encontram-se nos Estados do Piauí, do Ceará, do Maranhão e da Bahia, sendo que as tocas de pequenos animais (como roedores e marsupiais) e os sítios arqueológicos são os locais de maior risco de infecção pelo fungo (WANKE, 1994). Sabe-se que um caso de rinosporidiose ocorreu em égua Puro Sangue Inglês de três anos de idade. Nos outros casos não foram especificados o sexo, a raça e a idade dos animais. A rinosporidiose é causada pelo protista aquático Rhinosporidium seeberi, pertencente à classe Mesomycetozoea e anteriormente classificado como fungo (ARSECULERATNE, 2002; ARSECULERATNE, 2005; MENDOZA et al., 2005).

No exame histopatológico dos casos de pólipos, foi observada hiperplasia do epitélio (escamoso estratificado do vestíbulo e colunar pseudo-estratificado ciliado da porção respiratória da cavidade nasal) e proliferação de tecido conjuntivo fibrovascular (Figura 2) associado à presença de lesão inflamatória acentuada e difusa, com infiltrado mononuclear (composto principalmente por linfócitos

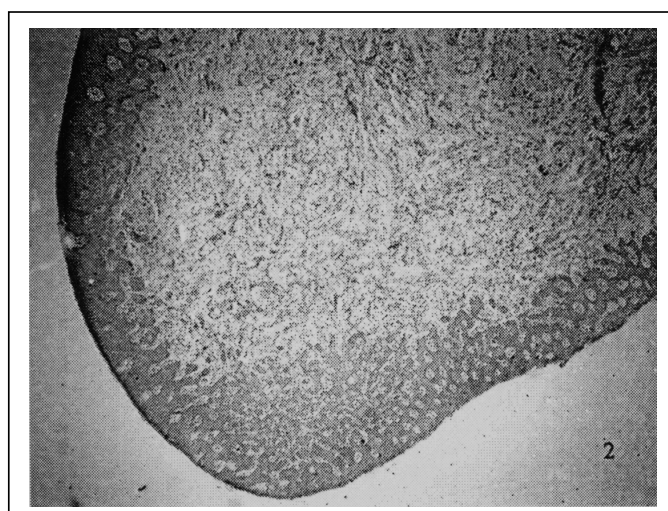

Figura 2 - Cavidade nasal. Pólipo nasal. Mula. Presença de hiperplasia do epitélio escamoso estratificado (do vestíbulo) e proliferação do tecido conjuntivo fibrovascular. HE, obj. $4 \mathrm{X}$. e plasmócitos) em quatro casos e exudato mucopurulento (composto principalmente por neutrófilos e piócitos) em dois casos. Não foi identificada a etiologia. O quadro observado estava de acordo com o descrito por outros autores, que também classificam os pólipos nasais como lesões tumoriformes de origem inflamatória não identificada (STICKLE \& JONES, 1976; WATT \& BECK, 1997; NICKELS, 1993; KNOTTENBELT \& PASCOE, 1994; JONES et al., 2000). Entretanto, diferentemente do relatado por WATT \& BECK (1997), não foram observados cistos. Nos animais com pólipos, não foram especificados o sexo, a raça e a idade. Sabe-se que ocorreram cinco casos em cavalos e um caso em mula.

No exame clínico do cavalo com amiloidose, foram encontradas duas lesões tumoriformes ulceradas, com crostas hemorrágicas e amareladas na mucosa do septo nasal. Uma das lesões possuía cerca de $2 \mathrm{~cm}$ de diâmetro e estava localizada na metade lateral direita da cavidade nasal. A outra media $1 \mathrm{~cm}$ de diâmetro e foi observada na metade lateral esquerda. Estas alterações foram diferentes das relatadas por KNOTTENBELT \& PASCOE (1994), que descreveram a lesão como placas firmes e pálidas. Os cortes histológicos dessas lesões, corados pela hematoxilina-eosina, apresentavam massas globulares e lineares constituídas por material hialino eosinofílico (Figura 3). Na coloração de vermelho-congo, este material apresentou-se laranjavermelho, confirmando tratar-se de amilóide. As alterações microscópicas foram semelhantes às relatadas na literatura (KNOTTENBELT \& PASCOE, 1994; JONES et al., 2000). O animal com amiloidose era uma égua da raça Quarto de Milha de dois anos de idade, porém não havia mais informações que

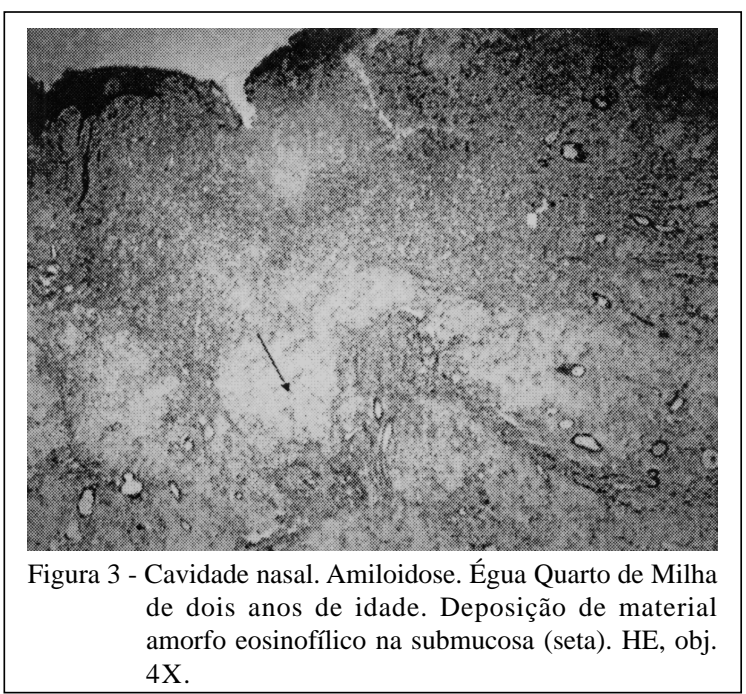


permitissem identificar a etiologia da doença nesse animal. A amiloidose em cavalos ocorre em animais com doenças inflamatórias crônicas e aqueles usados para a produção de soro hiperimune (KNOTTENBELT \& PASCOE, 1994), sendo o amilóide presente do tipo AA (JONES et al., 2000). Entretanto, nos casos de amiloidose nasal em cavalos, de etiologia desconhecida, e naqueles associados ao mieloma múltiplo, o amilóide é do tipo AL (DUNGWORTH, 1993).

No corte histológico, o hematoma etmoidal progressivo estava revestido por epitélio pseudoestratificado ciliado e tecido conjuntivo. O estroma apresentava seios revestidos de endotélio, contendo hemossiderófagos e sangue no interior (Figuras 4A e 4B). Observaram-se também células gigantes multinucleadas e depósitos granulares basofílicos compatíveis com cálcio. As alterações histopatológicas se apresentaram similares às descritas por outros autores (SPECHT et al., 1990; PASCOE, 1993; TATE, 2002). O animal com esta lesão era uma égua da raça Árabe de quatro anos de idade. A causa do hematoma etmóide é desconhecida e, na ausência de tratamento, que é preferencialmente cirúrgico, o seu prognóstico é desfavorável. Isso porque a doença é progressiva, podendo causar severa perda de sangue e obstrução nasal (NICKELS, 1993; KNOTTENBELT \& PASCOE, 1994). Vários carcinomas, no trato respiratório superior, podem apresentar sinais clínicos e aparência semelhante ao hematoma etmoidal (KNOTTENBELT \& PASCOE, 1994; TATE, 2002), o que ressalta a importância da histopatologia na determinação do diagnóstico final.

Apesar de neoplasias serem consideradas uma das causas comuns de lesões tumoriformes na cavidade nasal de cavalos, no presente estudo, nenhuma das alterações microscópicas observadas possuía aspecto neoplásico. Uma das mais freqüentes neoplasias da cavidade nasal dos cavalos é o carcinoma epidermóide, entretanto, outros tumores podem ocorrer, tais como sarcomas, linfossarcoma, carcinoma e adenocarcinoma, fibroma, fibropapiloma, condroma e osteoma (NICKELS, 1993). Estes fatos ressaltam a importância do exame histopatológico não só para a diferenciação de lesões tumoriformes na cavidade nasal de eqüinos, mas também para a classificação das diferentes neoplasias que podem ocorrer.

Como não há levantamentos sobre a ocorrência destas afecções no Brasil, não foi possível fazer uma comparação com os resultados do nosso estudo. O único estudo encontrado foi o de LONDERO et al. (1977), que revisaram e relataram 13 casos de rinosporidiose em cavalos, quatro em mulas e um em bovino.

\section{CONCLUSÕES}

Clinicamente, as lesões observadas na cavidade nasal foram consideradas tumoriformes. No exame histopatológico, nenhuma delas se revelou como neoplasia. Ao contrário, foram diagnosticadas quatro doenças de natureza infecciosa, inflamatória ou proliferativa benigna. Portanto, é fundamental a histopatologia para o diagnóstico definitivo de lesões tumoriformes na cavidade nasal de eqüídeos, permitindo adotar o tratamento específico para cada uma das doenças.

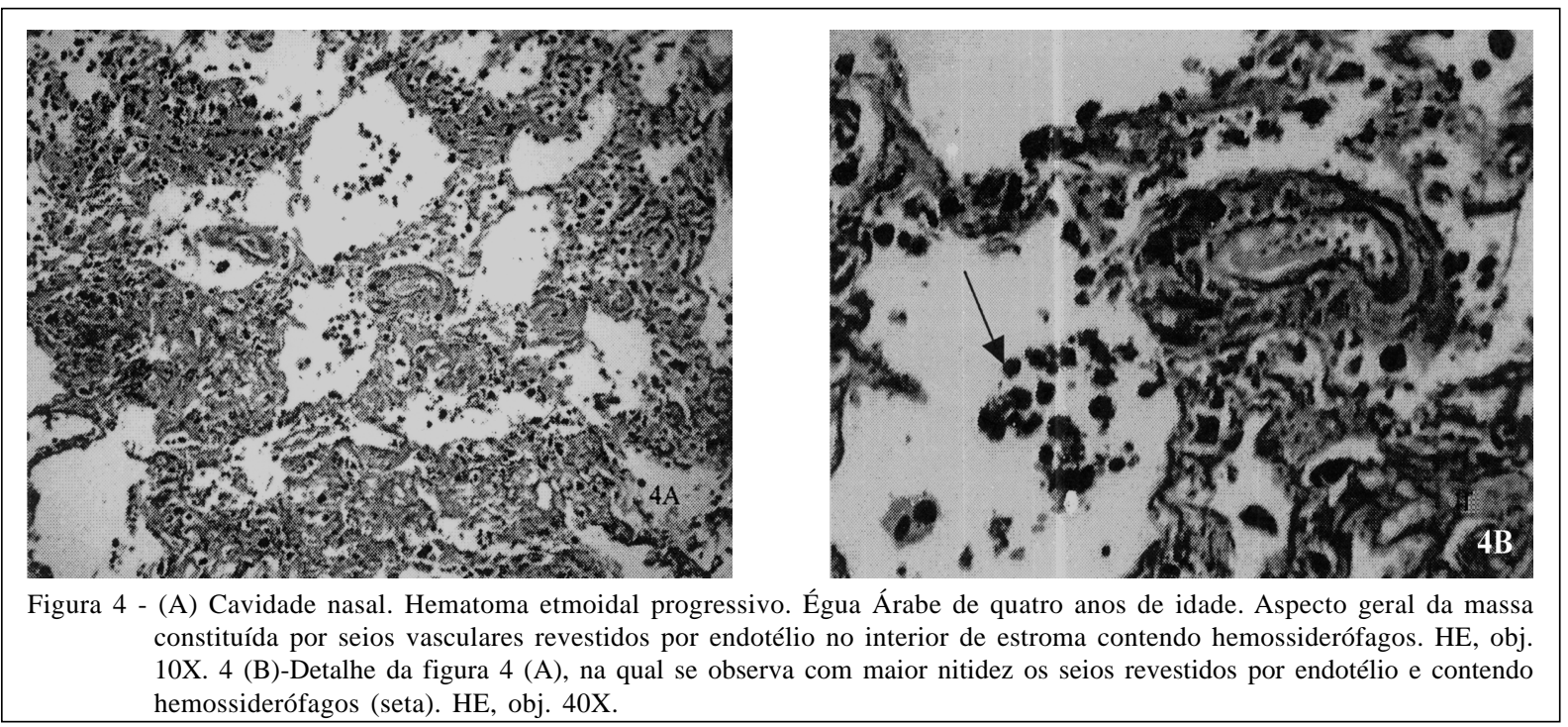

Ciência Rural, v.38, n.9, dez, 2008. 


\section{REFERÊNCIAS}

ARSECULERATNE, S.N. Recent advances in rhinosporidiosis and Rhinosporidium seeberi. Indian Journal of Medical Microbiology, v.20, n.3, p.119-131, 2002.

ARSECULERATNE, S.N. Rhinosporidiosis: what is the cause? Current Opinion in Infectious Diseases, v.18, n.2, p.113118, 2005.

BERROCAL, A.; LÓPEZ, A. Nasal rhinosporidiosis in a mule. Canadian Veterinary Journal, v.48, n.3, p.305-306, 2007.

DUNGWORTH, D.L. The Respiratory system. In: JUBB, K.V.F. et al. Pathology of domestic animals. San Diego: Academic, 1993. V.2, p.546-699.

JONES, T.C. et al. Patologia veterinária. 6.ed. São Paulo: Manole, 2000. 1415p.

KENNEDY, F.A. et al. Rhinosporidiosis: a description of an unprecedented outbreak in captive swans (Cygnus spp.) and a proposal for revision of the ontogenic nomenclature of Rhinosporidium seeberi. Journal of Medical and Veterinary Mycology, v.33, n.3, p.157-165, 1995.

KIM, D.Y. et al. Systemic AL amyloidosis associated with multiple myeloma in a horse. Veterinary Pathology, v.42, n.1, p.81-84, 2005.

KNOTTENBELT, D.C.; PASCOE R.R. Diseases and disorders of the horse. London: Mosby-Wolfe, 1994. 432p.

LONDERO, A.T. et al. Animal rhinosporidiosis in Brazil. Report of three additional cases. Mycopathologia, v.60, n.3, p.171-173, 1977.
MENDOZA, L. et al. Lacazia loboi and Rhinosporidium seeberi: a genomic perspective. Revista Iberoamericana de Micología, v.22, n.4, p.213-216, 2005.

MOISAN, P.G.; BAKER, S.V. Rhinosporidiosis in a cat. Journal of Veterinary Diagnostic investigation, v.13, n.4, p.352-354, 2001.

NICKELS, F.A. Diseases of the nasal cavity. Veterinary Clinics of North America: Equine Practice, v.9, n.1, p.111121, 1993.

PASCOE, J.R. Hematoma etmóide. In: SMITH, B.P. Tratado de Medicina Interna De Grandes Animais. São Paulo: Manole, 1993. V.1, p.566-569.

SPECHT, T.E. et al. Ethmoidal hematoma in nine horses. Journal of the American Veterinary Medical Association, v.197, n.5, p.613-616, 1990.

STICKLE, R.L.; JONES, R.D. Nasal polyp in a horse Veterinary Medicine Small Animal Clinician, v.71, n.10, p.1453-1456, 1976.

TATE, L.P. Noncontact free fiber ablation of equine progressive ethmoid hematoma. Clinical Techniques in Equine Practice, v.1, n.1, p.22-27, 2002.

WANKE, B. Coccidioidomicose. Revista da Sociedade Brasileira de Medicina Tropical, v.27, supl.IV, p.375-378, 1994.

WATT, B.C.; BECK, B.E. Removal of a nasal polyp in a standing horse. Canadian Veterinary Journal, v.38, n.2, p.108-109, 1997. 